OPEN ACCESS

Edited by:

Yanis Boumber,

Fox Chase Cancer Center.

United States

Reviewed by:

Janaki Deepak,

University of Maryland, Baltimore,

United States

Jacobus A. Burgers,

Antoni van Leeuwenhoek

Hospital, Netherlands

${ }^{*}$ Correspondence:

Serafeim Chrysikos makischr@hotmail.com

Specialty section: This article was submitted

to Thoracic Oncology,

a section of the journal

Frontiers in Oncology

Received: 14 November 2017

Accepted: 16 January 2018

Published: 30 January 2018

Citation:

Chrysikos S, Kaponi M, Triantafillidou C, Karampitsakos T,

Tzouvelekis A, Anyfanti M,

Marossis K, Konstantinou M,

Tringidou R, Bouros $D$ and Dimakou K (2018) A Rare Case of

Primary Intrapulmonary Neurilemmoma Diagnosed in a

43-Year-Old Asymptomatic

Man with a Well-defined

Intrapulmonary Mass.

Front. Oncol. 8:11

doi: 10.3389/fonc.2018.00011

\section{A Rare Case of Primary Intrapulmonary Neurilemmoma Diagnosed in a 43-Year-Old Asymptomatic Man with a Well- defined Intrapulmonary Mass}

\author{
Serafeim Chrysikos ${ }^{1 *}$, Maria Kaponi ${ }^{1}$, Christina Triantafillidou ${ }^{2}$, Theodoros Karampitsakos ${ }^{1}$, \\ Argyrios Tzouvelekis ${ }^{3}$, Maria Anyfanti ${ }^{1}$, Konstantinos Marossis ${ }^{1}$, Marios Konstantinou ${ }^{4}$, \\ Rodoula Tringidou ${ }^{5}$, Demosthenes Bouros ${ }^{3}$ and Katerina Dimakou ${ }^{1}$

\begin{abstract}
15th Pulmonology Department, Athens Chest Hospital "Sotiria", Athens, Greece, ${ }^{2} 6$ th Pulmonology Department, Athens Chest Hospital "Sotiria", Athens, Greece, " ${ }^{3}$ First Academic Department of Pneumonology, Athens Chest Hospital "Sotiria", Medical School, National and Kapodistrian University of Athens, Athens, Greece, " Thoracic Surgery Department, Athens Chest Hospital "Sotiria", Athens, Greece, ${ }^{5}$ Pathology Department, Athens Chest Hospital "Sotiria", Athens, Greece
\end{abstract}

Neurilemmoma (NL), also termed schwannoma, presents as a well-circumscribed and encapsulated mass in the human body and is almost always solitary. CT scan of a patient with NL shows a round, ovoid, or lobulated well-demarcated solid mass of soft tissue density. Primary intrathoracic neurogenic tumors location varies. However, the development of such tumors is by far more common in the costovertebral angle of the posterior mediastinum. Here, we report a rare case of a 43-year-old patient, never smoker and previously healthy, who presented with a mass adjacent to the right pulmonary hilum. This was an incidental finding on a chest X-ray after annual checkup at his workplace. The diagnosis was primary intrapulmonary NL. Primary intrapulmonary $\mathrm{NL}$ is an extremely rare tumor. However, based on the above, chest CT findings of a well-defined solid mass in an asymptomatic patient should raise the suspicion of $\mathrm{NL}$, irrespective of the tumor localization.

Keywords: intrapulmonary schwannoma, neurilemmoma, positron-emission tomography, tumor, mass

\section{INTRODUCTION}

Neurilemmoma (NL), also termed schwannoma, presents as a well-circumscribed and encapsulated mass in the human body and is almost always solitary. Primary nerve cell tumors of the lung are extremely rare, and they accounted for $0.2 \%$ of all pulmonary neoplasms (1). We herein report an interesting case of a 43-year-old patient of our clinic, who was diagnosed with $\mathrm{NL}$, after investigation of a mass adjacent to the right pulmonary hilum, which is a rare location for NL development.

\section{CASE PRESENTATION}

A 43-year-old man, previously healthy and never smoker presented with a mass adjacent to the right pulmonary hilum, found incidentally on a chest X-ray after a checkup conducted due to a change in his position at his workplace. He was completely asymptomatic and in excellent physical condition. 
Typical physical examination did not reveal abnormal findings (blood pressure 120/80 mmHg, pulses 72 beats per minute, temperature $36.8^{\circ} \mathrm{C}$ oxygen saturation $98 \%$ on $\mathrm{FiO}_{2} 21 \%$, lung auscultation without additional sounds). There were no palpable lymph nodes. Complete blood count and metabolic panel, urinalysis, and electrocardiography were normal. The tuberculin skin test was negative. Chest CT showed a small, well-defined, round, and solid lesion $(32 \mathrm{~mm} \times 27 \mathrm{~mm})$, in the superior segment of right lower lobe (Figure 1). Positron-emission tomography (PET) demonstrated a diffuse, low FDG uptake $\left(S_{U} V_{\max } 2.1\right)$ of the lesion, which exceeded that of vascular structure of the mediastinum (SUV $\mathrm{Sax}_{\max }$ 1.7) (Figure 2). Bronchoscopy was indicative of edema on the posterior wall of intermedius bronchus. Cytological examination from washing and brushing and endobronchial biopsy were negative for malignancy.

Given that the lesion exceeded that of vascular structure of the mediastinum, a discussion with the patient was conducted. Due to the fact that PET result was suspicious and the patient wanted a direct invasive procedure, the patient referred to a thoracic surgeon. Our working diagnosis was between a benign tumor

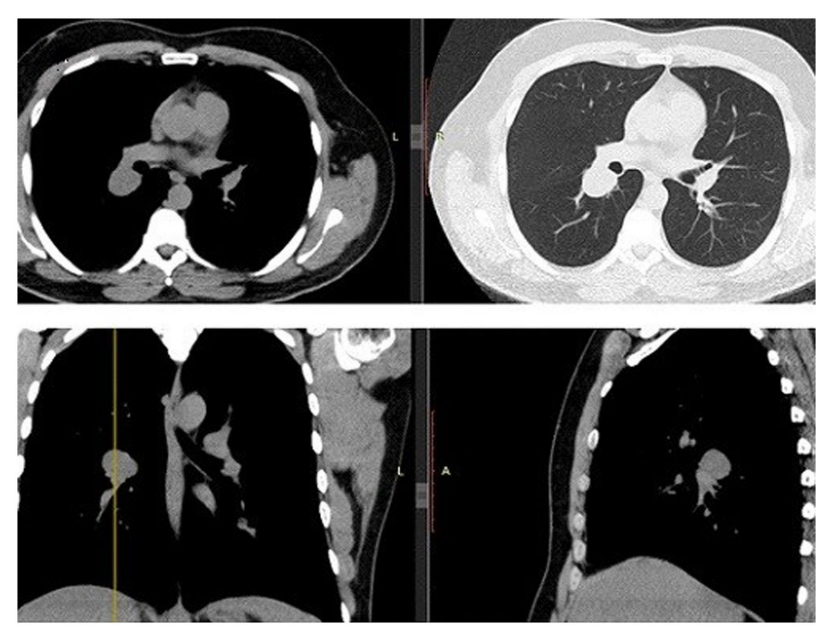

FIGURE 1 | Chest CT. Transverse/coronal/sagittal planes demonstrate a round homogenous mass $32 \mathrm{~mm} \times 27 \mathrm{~mm}$ in size in the superior segment of right lower lobe.

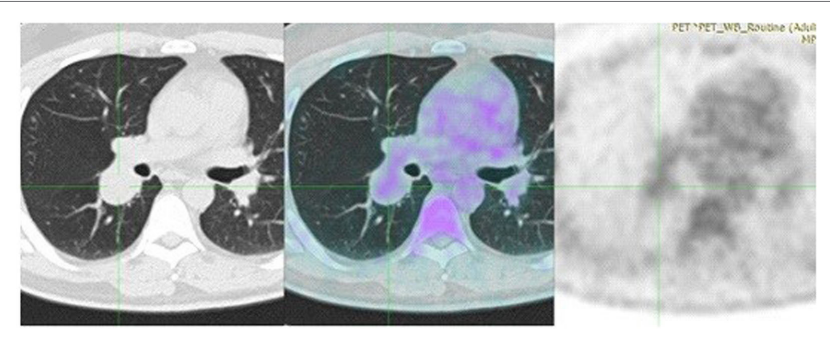

FIGURE 2 | Positron-emission tomography (PET) demonstrates a diffuse, low FDG uptake $\left(S U V_{\max } 2.1\right)$ of the lesion, which exceeded that of vascular structure of the mediastinum $\left(S \cup V_{\max }\right.$ 1.7). or a low grade neuroendocrine tumor. Patient underwent right thoracotomy with right lower lobectomy and right bronchoplasty of the bronchus intermedius. Post- surgery macroscopic examination validated that tumor was well demarcated, encapsulated and round, with $3 \mathrm{~cm}$ diameter. There was no evidence of invasion. The color of the cut surface was yellowish. Microscopic findings included elongated tumor cells with spindle-shaped nuclei and cellular palisading. There was no evidence of nuclear atypia or necrosis. Immunohistochemical staining was positive for S-100 protein and $\operatorname{EMA}(-), \operatorname{PANK}(-), \operatorname{CD} 68(-), \operatorname{SMA}(-)$, Desmine(-), CD31(-) (Figure 3). Interestingly, the diagnosis was primary pulmonary intrapulmonary NL (schwannoma). This was an extremely interesting finding as primary intrathoracic neurogenic tumors are most frequently developed in the costovertebral angle of the posterior mediastinum. As it was expected, the 2-year follow-up revealed no further abnormalities and the patient did not report any problem with regards to his quality of life.
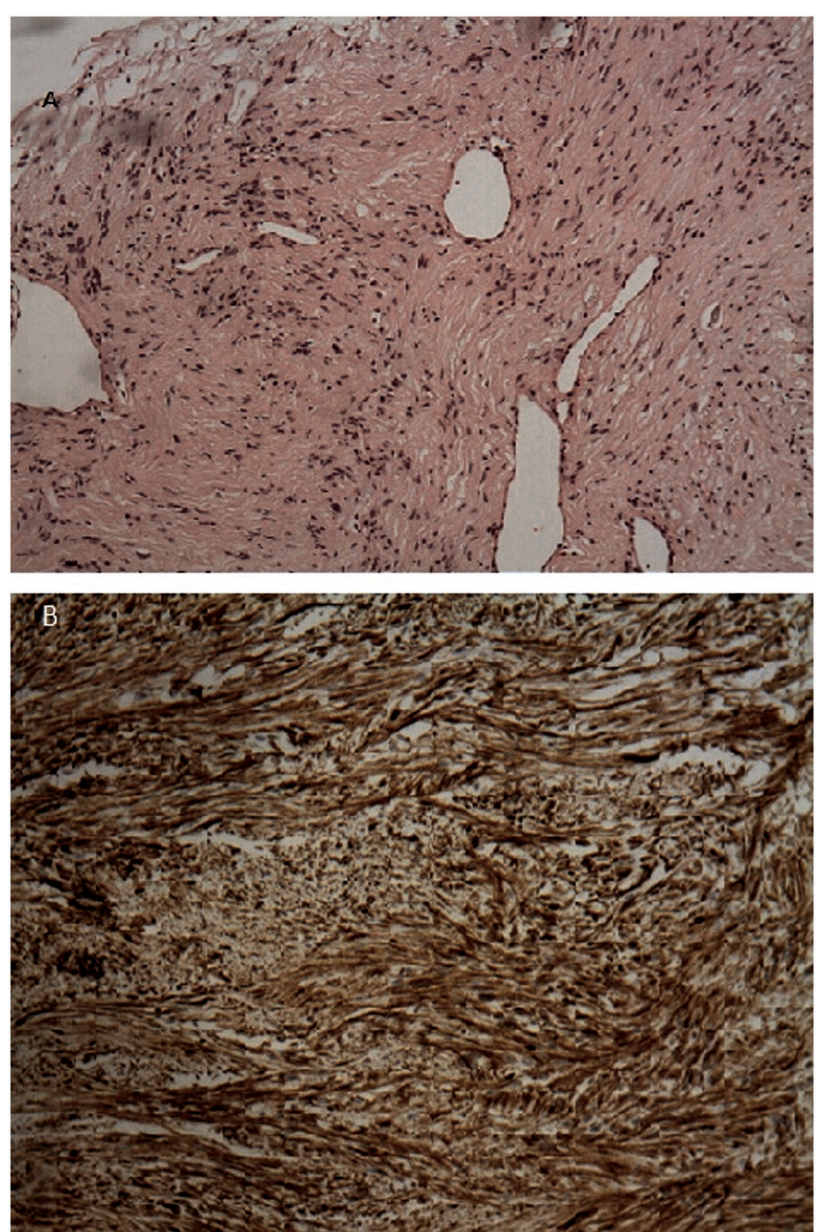

FIGURE 3 | (A) H-E stain revealed an area of encapsulated neoplasm surrounded by lung tissue. (B) Microscopic examination revealed proliferation of elongated tumor cells having spindle-shaped nuclei, with cellular palisading. No necrosis or nuclear atypia was observed.

Immunohistochemical staining demonstrated positive staining of the tumor cells for S-100 protein. 
Every part of the procedure was approved by the Institutional Board of Chest Hospital Sotiria, Athens, Greece. Written informed consent was obtained from the participant for the publication of this case report.

\section{DISCUSSION}

Neurilemmoma, also termed schwannoma, presents as a well circumscribed and encapsulated mass in the human body and is almost always solitary (1). Primary intrathoracic neurogenic tumors location varies. However, the development of such tumors is by far more common in the costovertebral angle of the posterior mediastinum (2). Primary nerve cell lung tumors represent an extremely rare entity. They arise from the autonomic nerve bundles of the blood vessels and derived from the cells of Schwann's sheath (3). Published case series of 62 patients ( 34 women) aged 5-83 years, with intrapulmonary or bronchial schwannomas concluded that the frequency of these tumors was $0.2 \%$ of all lung neoplasms $(1,4)$. Intrapulmonary schwannomas are centrally or peripherally located. Centrally located schwannomas are further subdivided into following two types: (i) intraluminal and (ii) extraluminal. Prognosis of schwannomas is good, as they represent benign, typically solitary tumors, with low rates of recurrence and malignant transformation. In addition, they are rarely multiple, and recurrence is uncommon $(5,6)$. Interestingly, there are reports of association between Von Recklinghausen's disease and primary neurogenic lung tumors $(2,7)$.

CT scan of a patient with NL shows a round, ovoid, or lobulated well demarcated, homogeneous, solid mass with density of soft tissue. Nonetheless, NL could also present on CT as partly solid mass due to hemorrhage or necrosis. Punctuate calcifications are often noted (6).

FDG-PET/CT is generally crucial for the differentiation between malignant and benign soft tissue lesions. Data derived from retrospective studies showed that the maximum standard uptake values ( $\mathrm{SUV}_{\max }$ ) of NL ranged from 1.3 to 6 (mean 3.2), while malignant neurogenic tumors (MPNST) had an uptake between 4.5 and 9.7 (mean 7.0) $(1,5,8,9)$. SUV values might vary widely owing to varying degrees of microvascular density, cellularity, and vascular permeability. Areas of necrosis or cystic changes are common reasons for heterogeneous uptake $(1,5,8,9)$.

Most patients with intrapulmonary NL present with no symptoms and mass is identified incidentally on the chest X-ray, as in our case. However, a considerable percentage of patients presents with symptoms mimicking asthma, bronchitis, or poststenotic pneumonia due to airway obstruction secondary to tumor (5).

Histologically, NL is typically sharply surrounded by a fibrous capsule and is associated with two microscopic patterns-the

\section{REFERENCES}

1. Yukawa T, Shimizu K, Hirami Y, Okita R, Saisho S, Maeda A, et al. A case report of intrapulmonary schwannoma. Gen Thorac Cardiovasc Surg (2014) 62:252-4. doi:10.1007/s11748-013-0233-5

2. Roviaro G, Montorsi M, Varoli F, Binda R, Cecchetto A. Primary pulmonary tumours of neurogenic origin. Thorax (1983) 38:942-5. doi:10.1136/ thx.38.12.942
Antoni A (cellular) and Antoni B (less cellular) (3, 4, 7). The former is associated with highly cellular areas of spindle-shaped cells interspersed with irregular, wave nuclei, and a palisade-like arrangement. The Antoni B pattern features include (i) relative hypocellularity, (ii) elongated cells with irregular fashion, (iii) separation of these cells from one another by matrix with poor or no staining for hematoxylin, eosin, and alcian blue stains. Strong, as well as diffuse staining for S-100 protein is a typical feature of these cells $(3,4,7)$. Differential diagnosis of histological presence of spindle cells includes NL, leiomyoma, fibroma, angiofibroma, and sclerosing hemangioma $(3,4,7)$. Therefore, immunohistochemical staining for Desmine and SMA (muscle origin tumors both smooth and skeletal), CD68 (histiocyte origin tumors), and CD31 (vascular origin tumors) is necessary $(3,4,7)$.

With regards to treatment of primary intrapulmonary $\mathrm{NL}$, successful surgical treatment using limited airway/sleeve resection, lobectomy, or rarely pneumonectomy for large or proximal lesions has been reported. The prognosis of intrapulmonary NL is very good, and to this end there are no reports of recurrence $(1,4,5,7)$.

\section{CONCLUSION}

Primary intrapulmonary NL is an extremely rare tumor; yet, chest CT findings of a well-defined solid mass in an asymptomatic patient should raise the suspicion of NL. There is no pathognomonic radiological feature, and PET scan usually shows low uptake values; yet, variable uptake values could be shown. Due to the low malignant potential of a primary intrapulmonary NL, tumor enucleation or partial lobectomy (if possible) is sufficient unless the tumor is more proximal, which may require lobectomy. Histologic examination with positive S-100 protein immunochemistry staining is required for the diagnosis.

\section{ETHICS STATEMENT}

This study was carried out in accordance with the recommendations of the journal, with written informed consent from the patient. The patient gave written informed consent in accordance with the Declaration of Helsinki. The protocol was approved by the Institutional Board of Chest Hospital Sotiria, Athens, Greece.

\section{AUTHOR CONTRIBUTIONS}

SC wrote the majority of the manuscript. TK wrote part of the manuscript and offered intellectual contribution. All the authors contributed to the results provided and approved the manuscript. $\mathrm{AT}, \mathrm{DB}$, and $\mathrm{KD}$ offered also intellectual contribution. 
6. Molloy C, Staples ED, Sriram PS. A 59-year-old man with a 10-cm lung mass. Chest (2008) 134:1088-91. doi:10.1378/chest.08-0607

7. Bosch X, Ramirez J, Font J, Bombi J, Ferrer J, Vendrell J, et al. Primary intrapulmonary benign schwannoma. A case with ultrastructural and immunohistochemical confirmation. Eur Respir J (1990) 3:234-7.

8. Nose H, Otsuka H, Otomi Y, Terazawa K, Takao S, Iwamoto S, et al. Correlations between F-18 FDG PET/CT and pathological findings in soft tissue lesions. J Med Invest (2013) 60:184-90. doi:10.2152/jmi.60.184

9. Beaulieu S, Rubin B, Djang D, Conrad E, Turcotte E, Eary JF. Positron emission tomography of schwannomas: emphasizing its potential in preoperative planning. AJR Am J Roentgenol (2004) 182:971-4. doi:10.2214/ajr.182.4. 1820971
Conflict of Interest Statement: The authors declare that the research was conducted in the absence of any commercial or financial relationships that could be construed as a potential conflict of interest.

Copyright $\odot 2018$ Chrysikos, Kaponi, Triantafillidou, Karampitsakos, Tzouvelekis, Anyfanti, Marossis, Konstantinou, Tringidou, Bouros and Dimakou. This is an open-access article distributed under the terms of the Creative Commons Attribution License (CC BY). The use, distribution or reproduction in other forums is permitted, provided the original author(s) and the copyright owner are credited and that the original publication in this journal is cited, in accordance with accepted academic practice. No use, distribution or reproduction is permitted which does not comply with these terms. 